\title{
Growth in a Turing Model of Cortical Folding
}

\author{
Gregory Toole*, Monica K. Hurdal* \\ * Department of Mathematics, Florida State University, Tallahassee, FL 32306-4510, USA \\ Emails: gtoole@math.fsu.edu, mhurdal@math.fsu.edu
}

Received: 12 July 2012, accepted: 25 September 2012, published: 15 October 2012

\begin{abstract}
The brain's cerebral cortex is folded into many gyri (hills) and sulci (valleys). Little is known about how the cortex folds or why the folds are located where they are. We have developed a spatio-temporal mathematical model of cortical folding to address this question. Our model utilizes a Turing reaction-diffusion system on an exponentially growing prolate spheroidal domain. This domain approximates the shape of the lateral ventricle (LV) during cortical development. The Intermediate Progenitor Model (IPM) of cortical folding states that regional patterning of self-amplication of intermediate progenitor cells (IPCs) in the subventricular zone of the $\mathrm{LV}$ corresponds with the formation of cortical folding. As selfamplication of IPCs is genetically controlled via chemical gradients, a Turing system is a logical choice to create a mathematical representation of the IPM. A growing domain model of cortical folding may be more realistic than previous static domain models of cortical folding since it incorporates the growth that naturally occurs as the brain develops. By comparing patterns generated by our growing prolate spheroid Turing system with those generated by a static prolate spheroid Turing system, we show that the addition of growth causes a significant change in system behavior; the system produces transient patterns instead of converging to one final pattern. Our model illustrates the importance of including growth in a model of cortical folding and can be utilized to explain certain human diseases of cortical folding.
\end{abstract}

Keywords-cortical folding; morphology; neurobiology; Turing system

\section{INTRODUCTION}

The cerebral cortex of the brain is folded into an intricate pattern of gyri (hills) and sulci (valleys). The pattern of cerebral cortical folds varies from species to species as well as between individuals of the same species. Current biological models that attempt to explain the underlying processes of cortical folding conflict with one another; some emphasize the role of physical tension created by axonal connections within the cortex, while others highlight the importance of genetic chemical factors that influence cortical cells and their precursors. Furthermore, it is extremely difficult to perform neuroscience experiments to investigate cortical folding in living humans. Because of this biological debate and lack of experimental data, we have created a spatio-temporal mathematical model of cortical folding patterns in the brain. Our model employs a Turing reaction-diffusion system on an exponentially growing prolate spheroidal domain. Turing systems have been used to mathematically model pattern formation in many different areas of biological development, such as zebra stripes, giraffe spots, and alligator tooth formation [13], [15].

Previous biomathematical models of cortical folding employed Turing systems on a static domain [26], failing to capture the growth of the organism that naturally occurs as development progresses. Our model employs a growing domain, allowing us to create a more biologically realistic model of cortical folding by incorporating developmental growth. Numerical simulations demonstrate that the inclusion of domain growth in a Turing system causes a fundamental change in the pattern-generating behavior of the system, causing it to generate transiently evolving patterns rather than one convergent pattern. Incorporating domain growth into a Turing system model of cortical folding also allows one 


\section{G. Toole et al., Growth in a Turing Model of Cortical Folding}

to model certain diseases of cortical folding.

\section{BIOLOGICAL BACKGROUND}

The ventricular system of the brain consists of four ventricles: the two lateral ventricles (LVs), the third ventricle, and the fourth ventricle [25]. The ventricular zone (VZ) lines the lateral wall of the LVs and contains special proliferative cells that play a role in cortical development [17]. Continuing outward from the inside of a $\mathrm{LV}$, one passes from the $\mathrm{VZ}$ to the subventricular zone (SVZ), another area containing proliferative cells implicated in cortical development [17]. According to the Intermediate Progenitor Model (IPM) of cortical folding [12], intermediate progenitor cells (IPCs) in the SVZ undergo self-amplifying cell division with the number of rounds of cell division varying regionally throughout the SVZ. IPCs then divide into neurons which populate the upper layers of the cortex. Areas of high IPC selfamplification lead to cortical areas highly populated with neurons, forming gyri, while areas of low IPC selfamplfication lead to cortical areas with fewer neurons, forming sulci. IPCs and hence cortical folding are regulated via a genetic chemical gradient; namely, Pax6 and Wnt have been shown to affect the number of IPCs and proper cortical development in mice [22].

Another important role of the SVZ in brain development is the production of a structure called the germinal matrix (GM), which is also located along the lateral wall of the LVs [1], [11]. The GM contains precursors of neurons and glial cells and has been observed to grow exponentially from 11 to 23 weeks gestational age (GA) [1], [11]. The period of GM exponential growth overlaps with a period of development during which cortical folding occurs in humans, as primary cortical folds in humans emerge from 10 weeks GA to 30 weeks GA [9].

\section{TURING SySTEMS}

Turing reaction-diffusion systems are activatorinhibitor systems originally created to model chemical gradient concentrations on the developing embryo [28]. Let $u(\mathbf{X}, t)$ and $v(\mathbf{X}, t)$ represent the concentrations at time $t \geq 0$ of an activator morphogen and an inhibitor morphogen which are interacting on a static domain parametrized by position vector $\mathbf{X}$. Then the canonical Turing system is

$$
\left.\begin{array}{l}
\frac{\partial u}{\partial t}=d_{u} \nabla^{2} u+f(u, v), \\
\frac{\partial v}{\partial t}=d_{v} \nabla^{2} v+g(u, v),
\end{array}\right\}
$$

where $0<d_{u}<d_{v}$ are the respective diffusion coefficients of $u$ and $v$ and $f, g$ are the reaction kinetics. If System (1) possesses a spatially uniform steady state $\left(u_{0}, v_{0}\right)$ which is linearly stable in the absence of diffusion but is driven unstable by noise when diffusion is present, then System (1) is capable of generating spatially inhomogeneous patterns. These two properties must be satisfied in order for System (1) to exhibit Turing systems' characteristic pattern-generating behavior [15], [28]. We refer to these properties as Turing criteria.

Since we wish to include developmental growth as a part of our model of cortical folding, a growing domain must be incorporated into System (1). To accomplish this, let $S_{t} \subset \mathbb{R}^{3}$ be a two-dimensional regular growing surface with position vector $\mathbf{X}=\mathbf{X}(\zeta, \eta, t)$, where $\zeta, \eta$ parametrize $S_{t}$ in space and $t \geq 0$. Let $u(\mathbf{X}, t)$ and $v(\mathbf{X}, t)$ be the concentrations of two chemical substances on $S_{t}$ with diffusion coefficients $D_{u}$ and $D_{v}$, respectively. If we define $D=D_{u} / D_{v}, h_{1}=\left|\mathbf{X}_{\zeta}\right|, h_{2}=$ $\left|\mathbf{X}_{\eta}\right|$, then System (1) becomes

$$
\left.\begin{array}{l}
u_{t}=D \Delta_{s} u-\partial_{t}\left(\ln \left(h_{1} h_{2}\right)\right) u+\omega f(u, v), \\
v_{t}=\Delta_{s} v-\partial_{t}\left(\ln \left(h_{1} h_{2}\right)\right) v+\omega g(u, v),
\end{array}\right\}
$$

where $\omega>0$ is the domain scale parameter and

$$
\Delta_{s} \phi=\frac{1}{h_{1} h_{2}}\left[\left(\frac{h_{2}}{h_{1}} \phi_{\zeta}\right)_{\zeta}+\left(\frac{h_{1}}{h_{2}} \phi_{\eta}\right)_{\eta}\right]
$$

is the Laplace-Beltrami operator on $S_{t}$ (with $\phi=$ $u, \phi=v)[21]$. Notice that the incorporation of domain growth into a Turing system results in a third term in each reaction-diffusion equation: $-\partial_{t}\left(\ln \left(h_{1} h_{2}\right)\right) \phi$ (for $\phi=u, \phi=v)$. This new term represents dilution of the $u, v$ concentrations due to the growth of the domain [21].

System (2) can construct a Turing system on any regular growing surface, but since the growing domain of interest for our model is an exponentially prolate spheroid, we shall incorporate prolate spheroidal coordinates into the system. A prolate spheroid is the result of rotating an ellipse about its major axis and can be defined by the prolate spheroidal coordinate system [8],

$$
\begin{aligned}
& x=\frac{f}{2} \sqrt{\left(1-\eta^{2}\right)\left(\xi^{2}-1\right)} \cos 2 \pi \zeta, \\
& y=\frac{f}{2} \sqrt{\left(1-\eta^{2}\right)\left(\xi^{2}-1\right)} \sin 2 \pi \zeta, \\
& z=\frac{f}{2} \eta \xi
\end{aligned}
$$

where $\xi>1$ controls domain shape via eccentricity, $\eta=$ $\cos \theta \in[-1,1]$ with polar angle $\theta, \zeta=\frac{\phi}{2 \pi} \in[0,1)$ with azimuthal angle $\phi$, and $f$ is the interfocal distance. We 


\section{G. Toole et al., Growth in a Turing Model of Cortical Folding}

define the position vector $\mathbf{X}$ on an exponentially growing prolate spheroid as

$\mathbf{X}(\zeta, \eta, t)=\rho(t)\left(\begin{array}{c}\frac{f_{0}}{2} \sqrt{\left(\xi^{2}-1\right)\left(1-\eta^{2}\right)} \cos 2 \pi \zeta \\ \frac{f_{0}}{2} \sqrt{\left(\xi^{2}-1\right)\left(1-\eta^{2}\right)} \sin 2 \pi \zeta \\ \frac{f_{0}}{2} \xi \eta\end{array}\right)$,

where $f_{0}$ is the interfocal distance at $t=0$ and $\rho(t)=$ $e^{R t}$ is the growth function with growth rate $R>0$. Using Equation (4), the Laplace-Beltrami operator given in Equation (3) becomes

$$
\begin{aligned}
\Delta_{s}= & \frac{1}{\pi^{2} \rho^{2} f_{0}^{2}\left(1-\eta^{2}\right)\left(\xi^{2}-1\right)} \phi_{\zeta \zeta} \\
& +\frac{4\left(1-\eta^{2}\right)}{\rho^{2} f_{0}^{2}\left(\xi^{2}-\eta^{2}\right)} \phi_{\eta \eta}-\frac{4 \eta\left(2 \xi^{2}-\eta^{2}-1\right)}{\rho^{2} f_{0}^{2}\left(\xi^{2}-\eta^{2}\right)^{2}} \phi_{\eta},
\end{aligned}
$$

and the dilution term reduces to

$$
-\partial_{t}\left(\ln \left(h_{1} h_{2}\right)\right) \phi=-2 \frac{\dot{\rho}}{\rho} \phi=-2 R \phi .
$$

Overall, System (2) on an exponentially growing prolate spheroidal domain becomes

$$
\left.\begin{array}{l}
u_{t}=D \Delta_{s} u-2 R u+\omega f(u, v), \\
v_{t}=\Delta_{s} v-2 R v+\omega g(u, v),
\end{array}\right\}
$$

where $u=u(\zeta, \eta, t)$ and $v=v(\zeta, \eta, t)$.

\section{TURING CONDITIONS}

Next, we derive mathematical conditions whose satisfaction ensures that System (5) satisfies the two Turing criteria and thus can generate patterns. These mathematical conditions are called Turing conditions and are derived using linear stability analysis in the method of [10].

\section{A. Turing Criterion: Linear Stability}

To begin, we rewrite System (5) as

$$
\left.\begin{array}{l}
u_{t}=\frac{D}{\rho^{2}} \Delta_{\dagger} u-2 R u+\omega f(u, v), \\
v_{t}=\frac{1}{\rho^{2}} \Delta_{\dagger} v-2 R v+\omega g(u, v),
\end{array}\right\}
$$

where $\Delta_{\dagger}=\rho^{2} \Delta_{s}$. Let $\left(u_{0}, v_{0}\right)$ be a spatially uniform steady state of System (5) which remains a steady state in both the presence and absence of diffusion; in other words,

$$
\begin{aligned}
& 0=-2 R u_{0}+\omega f\left(u_{0}, v_{0}\right), \\
& 0=-2 R v_{0}+\omega g\left(u_{0}, v_{0}\right) .
\end{aligned}
$$

If $\mathbf{w}(t)=\left(\begin{array}{l}u(t)-u_{0} \\ v(t)-v_{0}\end{array}\right)=\left(\begin{array}{c}\epsilon_{u} \\ \epsilon_{v}\end{array}\right)$ is defined to be a perturbation from $\left(u_{0}, v_{0}\right)$, then System (6) can be rewritten as

$$
\begin{aligned}
\mathbf{w}_{t} & =\left(\begin{array}{c}
u_{t} \\
v_{t}
\end{array}\right) \\
& =\left(\begin{array}{l}
-2 R\left(u_{0}+\epsilon_{u}\right)+\omega f\left(u_{0}+\epsilon_{u}, v_{0}+\epsilon_{v}\right) \\
-2 R\left(v_{0}+\epsilon_{v}\right)+\omega g\left(u_{0}+\epsilon_{u}, v_{0}+\epsilon_{v}\right)
\end{array}\right) .
\end{aligned}
$$

Performing a Taylor expansion of $u_{t}, v_{t}$ around $\left(u_{0}, v_{0}\right)$ allows us to write

$$
\begin{aligned}
& u_{t} \approx-2 R \epsilon_{u}+\omega\left[\epsilon_{u} f_{u}\left(u_{0}, v_{0}\right)+\epsilon_{v} f_{v}\left(u_{0}, v_{0}\right)\right], \\
& v_{t} \approx-2 R \epsilon_{v}+\omega\left[\epsilon_{u} g_{u}\left(u_{0}, v_{0}\right)+\epsilon_{v} g_{v}\left(u_{0}, v_{0}\right)\right],
\end{aligned}
$$

from which it follows that

$$
\mathbf{w}_{t}=-2 R \mathbf{w}+\omega A \mathbf{w},
$$

where $A=\left(\begin{array}{ll}f_{u} & f_{v} \\ g_{u} & g_{v}\end{array}\right)_{\left(u_{0}, v_{0}\right)}$.

Next, consider solutions to Equation (7) with form $\mathbf{w}(t)=\mathbf{c} e^{\lambda t}$. In order to achieve linear stability of $\left(u_{0}, v_{0}\right)$, it must follow that $\mathbf{w} \rightarrow \mathbf{0}$ as $t \rightarrow \infty$. Hence, $\lambda$ must satisfy $\operatorname{Re}(\lambda)<0$. Substituting $\mathbf{w}(t)=\mathbf{c} e^{\lambda t}$ into Equation (7) and simplifying yields the eigenvalue equation

$$
\lambda \mathbf{c}=\tilde{A} \mathbf{c},
$$

where

$$
\tilde{A}=\omega A-2 R I .
$$

Solving for the characteristic polynomial in $\lambda$ and using the quadratic formula implies that $\operatorname{Re}(\lambda)<0$ when

$$
\begin{aligned}
\operatorname{tr} \tilde{A} & =\omega\left(f_{u}+g_{v}\right)-4 R<0 \text { and } \\
\operatorname{det} \tilde{A} & =\omega^{2}\left(f_{u} g_{v}-f_{v} g_{u}\right)-2 R \omega\left(f_{u}+g_{v}\right)+4 R^{2}>0 .
\end{aligned}
$$

These two inequalities constitute the first two Turing conditions for System (5).

\section{B. Turing Criterion: Diffusion-Driven Instability}

Linearizing System (6) about $\left(u_{0}, v_{0}\right)$ gives

$$
\mathbf{w}_{t}=D_{M} \Delta_{\dagger} \mathbf{w}-2 R \mathbf{w}+\omega A \mathbf{w},
$$

where $D_{M}=\frac{1}{\rho^{2}}\left(\begin{array}{ll}D & 0 \\ 0 & 1\end{array}\right)$. Consider solutions to Equation (8) with form

$$
\mathbf{w}(\mathbf{X}, t)=\sum_{k} c_{k} e^{\lambda t} \mathbf{Y}_{k}(\mathbf{X}),
$$




\section{G. Toole et al., Growth in a Turing Model of Cortical Folding}

where $\mathbf{Y}_{k}$ are prolate sheroidal harmonics. Since $\mathbf{Y}_{k}$ satisfy $\Delta_{\dagger} \mathbf{Y}_{k}=-k^{2} \mathbf{Y}_{k}$, substituting Equation (9) into Equation (8) and simplifying gives

$$
\sum_{k} c_{k}\left(\lambda \mathbf{Y}_{k}+D_{M} k^{2} \mathbf{Y}_{k}+2 R \mathbf{Y}_{k}-\omega A \mathbf{Y}_{k}\right)=0
$$

For nontrivial solutions $\mathbf{w}$ it must be that $c_{k} \neq 0$. It then follows that

$$
\lambda \mathbf{Y}_{k}=\left(-D_{M} k^{2}-2 R I+\omega A\right) \mathbf{Y}_{k},
$$

which is another eigenvalue equation. Nontrivial w occur when $\operatorname{det}\left(\tilde{A}-D_{M} k^{2}-\lambda I\right)=0$, and evaluating this determinant yields

$$
\lambda^{2}+\lambda\left[\frac{k^{2}}{\rho^{2}}(1+D)-\operatorname{tr} \tilde{A}\right]+h\left(k^{2}\right)=0,
$$

where

$$
\begin{aligned}
h\left(k^{2}\right)= & \frac{D}{\rho^{4}}\left(k^{2}\right)^{2}+\operatorname{det} \tilde{A} \\
& +\frac{k^{2}}{\rho^{2}}\left[2 R(1+D)-\omega\left(f_{u}+D g_{v}\right)\right] .
\end{aligned}
$$

Recalling that $\mathbf{w}(\mathbf{X}, t)=\sum_{k} c_{k} e^{\lambda t} \mathbf{Y}_{k}(\mathbf{X})$, it follows that diffusion-driven instability of $\left(u_{0}, v_{0}\right)$ occurs when $\operatorname{Re}(\lambda)>0$. Solving Equation (10) for $\lambda$ tells us that $\operatorname{Re}(\lambda)>0$ when

$$
\begin{aligned}
& 2 R(1+D)-\omega\left(f_{u}+D g_{v}\right)<0 \text { and } \\
& R^{2}\left[4-\frac{(1+D)^{2}}{D}\right]+\omega^{2}\left(f_{u} g_{v}-f_{v} g_{u}\right) \\
& \quad+R \omega\left[\frac{1}{D}(1+D)\left(f_{u}+D g_{v}\right)-2\left(f_{u}+g_{v}\right)\right] \\
& \quad<\frac{\omega^{2}}{4 D}\left(f_{u}+D g_{v}\right)^{2} .
\end{aligned}
$$

These two inequalities constitute the final two Turing conditions for System (5).

\section{THe MOdel}

Our mathematical model of cortical folding utilizes System (5), a Turing reaction-diffusion system on an exponentially growing prolate spheroidal domain. We select an exponentially growing prolate spheroidal domain to represent the LV and use the surface of the domain to represent the SVZ. Patterns generated by the model's Turing system represent regions of activation and nonactivation for self-amplification of IPCs in the SVZ; this regional self-amplification of IPCs then leads to cortical folds as described by the IPM.

Since Turing systems were originally created to model chemical morphogen concentration gradient patterns, they are useful for modeling developmental phenomena involving patterns of genetic chemical gradients. As mentioned in Section II], evidence suggests that genes regulate cortical folding via a chemical gradient, making a Turing system a reasonable choice for a mathematical model of cortical folding.

Recall that the GM grows exponentially over a period of development during which cortical folds are formed. Since the GM is produced by the SVZ and the SVZ is the site of self-amplification of IPCs (a key factor in the IPM), an exponentially growing domain is a reasonable choice for our model.

Neurogenesis in humans occurs approximately during embryonic days 43 to 120 [23]. Early in neurogenesis, the cerebral hemispheres are prolate spheroidal in shape, with the LVs accounting for almost all of the cerebral hemispheres' volume [26]. Thus, the LVs at this time of development are also prolate spheroidal in shape, making a prolate spheroidal domain a reasonable choice for our model.

To fully define our model's Turing system given in System (5), we must select reaction kinetics functions $f(u, v), g(u, v)$. We select nondimensional Barrio-VareaMaini (BVM) kinetics [4],

$$
\begin{aligned}
& f(u, v)=u+a v-C u v-u v^{2}, \\
& g(u, v)=b v+h u+C u v+u v^{2},
\end{aligned}
$$

so that System (5) becomes

$$
\left.\begin{array}{l}
u_{t}=D \Delta_{s} u-2 R u+\omega\left(u+a v-C u v-u v^{2}\right), \\
v_{t}=\Delta_{s} v-2 R v+\omega\left(b v+h u+C u v+u v^{2}\right) .
\end{array}\right\}
$$

BVM kinetics are phenomenological and are not modeled after any particular physical or chemical mechanism. Since the underlying mechanism of cortical folding is not fully understood, BVM kinetics are an appropriate choice for our model.

\section{A. Numerical Results}

To observe the patterns produced by System (11), we performed numerical simulations using a forward time, central space finite difference scheme [14]. Kinetics parameters were selected from the literature and were as follows:

$$
D=0.516, a=1.112, b=-1.01, C=0 .
$$

Initial conditions consisted of random values $\phi \in$ $[-0.5,0.5]$ along the domain's equator; other points on the domain were initialized as zero. The random initial values were seeded due to Turing systems' intrinsic high 

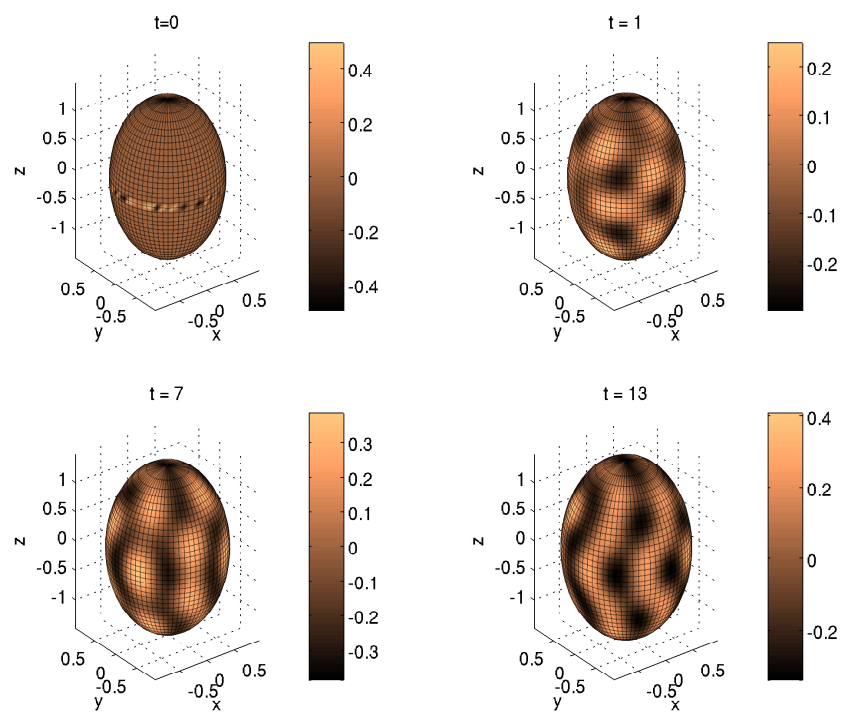
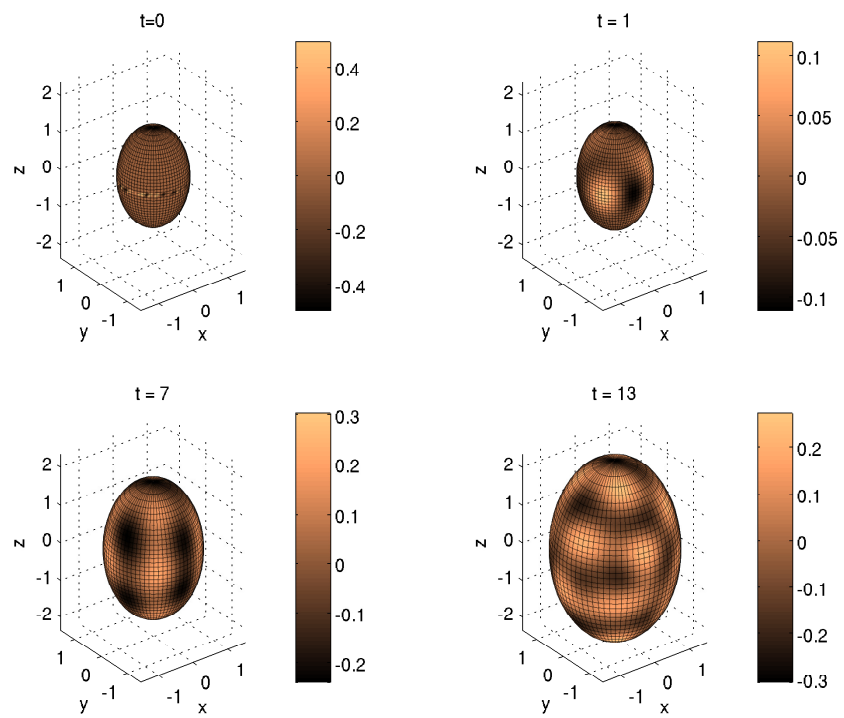

Fig. 1. Transient patterns generated by System (11) with $R=0.009$, $\omega=150$.
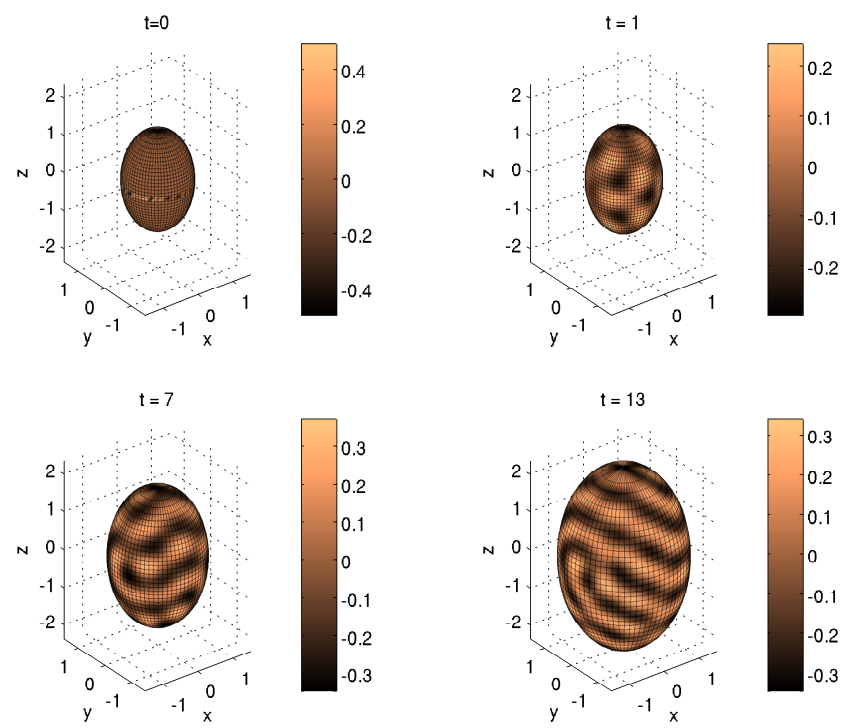

Fig. 2. Transient patterns generated by System (11) with $R=0.045$, $\omega=150$.

sensitivity to initial conditions [29]. Initial interfocal distance was chosen to be $f_{0}=2$ and domain shape was fixed by fixing $\xi$ at $\xi=1.3141$, giving a domain with initial surface area $4 \pi$, identical to that of the unit sphere. Simulations were varied only by altering the growth rate parameter $R$ and the domain scale parameter $\omega$.

System (11) generated transient patterns that constantly evolve from one pattern to another as elapsed time $t$ progresses (See Figures 113). As these transient patterns evolve, the number of stripes or spots in the pattern increases with increasing $t$. The pattern-
Fig. 3. Transient patterns generated by System 11 with $R=0.045$, $\omega=60$.

generating behavior of our growing domain system sharply contrasts with that of a static domain Turing system, which eventually converges to one final pattern. It is therefore clear that the addition of growth to a Turing system causes a major change in the system's pattern-generating behavior. Increasing the value of $R$ or $\omega$ causes System (11) to generate a more complex pattern (more stripes or spots) at a given $t$ (compare Figure 1 with Figure 2 and compare Figure 2 with Figure 3 ). Increasing these parameters also increased the frequency of transient pattern change; that is, the system evolved from pattern to pattern more quickly.

\section{B. Application to Diseases of Cortical Folding}

1) Polymicrogyria: Polymicrogyria (PMG) is a disease of cortical folding in which the cortex is excessively folded into many small folds [2]. Common symptoms of PMG include mental retardation, epilepsy, and developmental delay [2]. Several different forms of PMG are associated with enlarged LVs, such as megalencephaly PMG with polydactyly and hydrocephalus (MPPH) [7], [20], unilateral PMG [19], and bilateral frontoparietal PMG (BFPP) [6], [27]. Our model can encapsulate the enlargement of LVs by increasing the growth rate $R$, leading to a larger prolate spheroid (and hence LV) at any $t>0$. Increasing the growth rate $R$ leads to smaller and more numerous stripes in the pattern produced by System (11) (see Figure 2). By interpreting the stripes as locations and sizes of cortical folds (as described in Section $\mathrm{V}$, this change in size and number of stripes 


\section{G. Toole et al., Growth in a Turing Model of Cortical Folding}

represents an increased number of small cortical folds, agreeing with the characteristic brain abnormalities of PMG.

2) Norman-Roberts Syndrome: Norman-Roberts Syndrome (NRS) is a rare, potentially fatal congenital disease affecting brain development [16], [18], [24]. The genetic basis and pathophysiology of NRS are not fully known. Symptoms of NRS include reduced head growth rate, microcephaly, type I lissencephaly (too few cortical folds), epilepsy, and mental retardation [3], [5], [16], [24]. Since NRS is associated with microcephaly, it is reasonable to assume that the smaller-than-normal NRS brain also has smaller-than-normal LVs. We thus model NRS by decreasing $R$ so that the domain representing the LV is smaller at any $t>0$. Decreasing $R$ leads to larger and less numerous stripes in the pattern generated by System (11) (see Figure 1). By once again interpreting the stripes as cortical folds, this change in size and number of stripes represents a decrease in the number of cortical folds, reproducing the distinguishing brain anomalies of NRS.

\section{CONCLUSION}

We have shown that it is important to consider growth when constructing a biomathematical model of cortical folding, as adding growth to our Turing system model of cortical folding not only makes it more biologically realistic but also significantly changes the system's patterngenerating behavior. Furthermore, by appropriately altering the domain growth rate, a growing domain Turing system model of cortical folding can model certain diseases of cortical folding.

\section{REFERENCES}

[1] J.A. Anstrom, C.R. Thore, D.M. Moody, V.R. Challa, S.M. Block, and W.R. Brown,"Germinal matrix cells associate with veins and a glial scaffold in the human fetal brain", Dev. Brain Res., vol. 160, pp. 96-100, 2005. http://dx.doi.org/10.1016/j.devbrainres.2005.07.016

[2] A.J. Barkovich, "Current concepts of polymicrogyria", Neuroradiology, vol. 52, pp. 479-487, 2010. http://dx.doi.org/10.1007/s00234-009-0644-2

[3] A.J. Barkovich, R.I. Kuzniecky, G.D. Jackson, R. Guerrini, and W.B. Dobyns, "A developmental and genetic classification for malformations of cortical development", Neurology, vol. 65, pp. 1873-1887, 2005. http://dx.doi.org/10.1212/01.wnl.0000183747.05269.2d

[4] R.A. Barrio, C. Varea, J.L. Aragon, and P.K. Maini. "A twodimensional numerical study of spatial pattern formation in interacting Turing systems", B Math. Biol., vol. 61 No. 3, pp. 483-505, 1999.

http://dx.doi.org/10.1006/bulm.1998.0093
[5] H. Caksen, O. Tuncer, E. Kirimi, J.P. Fryns, A. Uner, O. Unal, A. Cinal, and D. Odabas, "Report of two Turkish infants with Norman-Roberts syndrome", Genet. Counsel, vol. 15 No. 1, pp. 9-17, 2004.

[6] B.S. Chang, X. Piao, A. Bodell, L. Basel-Vanagaite, R. Straussberg, W.B. Dobyns, B. Qasrawi, R.M. Winter, A.M. Innes, T. Voit, P.E. Grand, A.J. Barkovich, and C.A. Walsh, "Bilateral frontoparietal polymicrogyria: Clinical and radiological features in 10 families with linkage to chromosome 16", Ann. Neurol., vol. 53, pp. 596-606, 2003. http://dx.doi.org/10.1002/ana.10520

[7] M. Colombani, M. Chouchane, G. Pitelet, L. Morales, P. Callier, J.P. Pinard, L. Lion-Francois, C. Thauvin-Robinet, F. Mugneret, F. Huet, L. Guibaud, and L. Faivre., "A new case of megalencephaly and perisylvian polymicrogyria with post-axial polydactyly and hydrocephalus: MPPH syndrome”, Eur. J. Med. Genet., vol. 49, pp. 466-471, 2006. http://dx.doi.org/10.1016/j.ejmg.2006.05.001

[8] K. Flammer, Spheroidal Wave Functions, Stanford University Press, Palo Alto, 1957.

[9] C. Garel, E. Chantrel, H. Brisse, M. Elmaleh, D. Luton, J.F. Oury, G. Sebag, and M. Hassan, "Fetal cerebral cortex: Normal gestational landmarks identified using prenatal MR imaging", Am. J. Neuroradiol., vol. 22, pp. 184-189, 2001.

[10] J. Gjorgjieva and J. Jacobsen, "Turing patterns on growing spheres: The exponential case", Discrete Cont. Dyn. S. Suppl. 2007, pp. 436-445, 2007.

[11] Y. Kinoshita, T. Okudera, E. Tsuru, and A. Yokota, "Volumetric analysis of the germinal matrix and lateral ventricles performed using MR images of postmortem fetuses", Am. J. Neuroradiol., vol. 22, pp. 382-388, 2001.

[12] A. Kriegstein, S. Noctor, and V. Martinez-Cerdeno, "Patterns of neural stem and progenitor cell division may underlie evolutionary cortical expansion", Nat. Rev. Neurosci., vol. 7 No. 11, pp. 883-890, 2006. http://dx.doi.org/10.1038/nrn2008

[13] P.M. Kulesa, G.C. Cruywagen, S.R. Lubkin, P.K. Maini, J. Sneyd, M.W.J. Ferguson, and J.D. Murray, "On a model mechanism for the spatial patterning of teeth primordia in the alligator", J. Theor. Biol., vol. 180, pp. 287-296, 1996. http://dx.doi.org/10.1006/jtbi.1996.0103

[14] K.W. Morton and D.F. Mayers, Numerical Solution of Partial Differential Equations: An Introduction, Cambridge, Cambridge, UK, 2nd edition, 2005.

[15] J.D. Murray, Mathematical Biology II, Springer, New York, 3rd edition, 2003.

[16] F. Natacci, M.F. Bedeschi, A. Righini, F. Inverardi, T. Rizzuti, C. Boschetto, F. Triulzi, R. Spreafico, C. Frassoni, and F. Lalatta, "Norman-Roberts syndrome: Characterization of the phenotype in early fetal life", Prenatal Diag., vol. 27, pp. 568572, 2007. http://dx.doi.org/10.1002/pd.1728

[17] S.C. Noctor, V. Martinez-Cerdeno, L. Ivic, and A.R. Kriegstein, "Cortical neurons arise in symmetric and asymmetric division zones and migrate through specific phases", Nat. Neurosci., vol. 7 No. 2, pp. 136-144, 2004. http://dx.doi.org/10.1038/nn1172

[18] M.G. Norman, M. Roberts, J. Sirois, and L.J.M. Tremblay, "Lissencephaly", Can. J. Neurol. Sci., vol. 3, pp. 39-46, 1976.

[19] I. Pascual-Castroviejo, S.I. Pascual-Pascual, J. Viano, V. Martinez, and R. Palencia, "Unilateral polymicrogyria: A common cause of hemiplegia of prenatal origin", Brain Dev.-Jpn, vol. 
23, pp. 216-222, 2001. http://dx.doi.org/10.1016/S0387-7604(01)00211-X

[20] T. Pisano, M. Meloni, C Cianchetti, M. Falchi, A. Nucaro, and D. Pruna, "Megalencephaly, polymicrogyria, and hydrocephalus MPPH syndrome: A new case with syndactyly", J. Child Neurol., vol. 23, pp. 916-918, 2008. http://dx.doi.org/10.1177/0883073808315623

[21] R.G. Plaza, F. Sanchez-Garduno, P. Padilla, R.A. Barrio, and P.K. Maini, "The effect of growth and curvature on pattern formation", J. Dyn. Differ. Equ, vol. 16 No. 4, pp. 1093-1121, 2004.

http://dx.doi.org/10.1007/s10884-004-7834-8

[22] A. Pontious, T. Kowalczyk, C. Englund, and R.F. Hevner, "Role of intermediate progenitor cells in cerebral cortex development", Dev. Neurosci-Basel, vol. 30, pp. 24-32, 2008. http://dx.doi.org/10.1159/000109848

[23] P. Rakic, "A small step for the cell, a giant leap for mankind a hypothesis of neocortical expansion during evolution", Trends Neurosci., vol. 18, pp. 383-388, 1995.

http://dx.doi.org/10.1016/0166-2236(95)93934-P

[24] C. Sergi, S. Zoubaa, and M. Schiesser, "Norman-Roberts syndrome: Prenatal diagnosis and autopsy findings", Prenatal Diag., vol. 20, pp. 505-509, 2000. http://dx.doi.org/10.1002/ 1097-0223(200006)20:6〈505::AID-PD850>3.0.CO;2-\#

[25] L. Sherwood, Human Physiology: From Cells to Systems, Thomson Brooks/Cole, Belmont, 5th edition, 2004.

[26] D.A. Striegel and M.K. Hurdal, "Chemically based mathematical model for development of cerebral cortical folding patterns", PLoS Comput. Biol., vol. 5 No. 9, e1000524, 2009. http://dx.doi.org/10.1371/journal.pcbi.1000524

[27] L. Sztriha and M. Nork, "Bilateral frontoparietal polymicrogyria and epilepsy”, Pediatr. Neurol., vol. 22, pp. 240-243, 2000. http://dx.doi.org/10.1016/S0887-8994(99)00142-3

[28] A.M. Turing, "The chemical basis of morphogenesis", Philos. T. Roy. Soc. B, vol. 237, pp. 37-72, 1952.

[29] C. Venkataraman, T. Sekimura, E.A. Gaffney, P.K. Maini, and A. Madzvamuse, "Modeling parr-mark pattern formation during the early development of Amago trout", Phys. Rev. E, vol. 84, 041923, 2011.

http://dx.doi.org/10.1103/PhysRevE.84.041923

